\title{
Spontaneous rupture of esophagus (Boerhaave's syndrome): Case report and literature review
}

MICHAEL J. ZAWISZA, DO

ALAN GEISLER, DO
The diagnosis of Boerhaave's syndrome, or spontaneous rupture of the esophagus, often is delayed because of its ambiguous presentation, which can mimic other acute medical emergencies. Exclusion of the syndrome from the initial differential diagnosis of the patient presenting with acute onset of chest or abdominal pain can mislead the clinician in his choice of therapy. Almost 90\% mortality has been seen in cases not treated with surgical repair within the first 48 hours after presentation. Rarely, reports of patient management and survival without surgical intervention have been reported; however, surgery is the treatment of choice. This paper discusses a case of Boerhaave's Syndrome with a prolonged delay (approximately 96 hours) between the initial insult and the surgical repair. Supportive care with intravenous fluids, nasogastric tube drainage and NPO status were responsible for the patient's survival during the interval and the successful outcome of the case following surgery.

Spontaneous esophageal rupture, or Boerhaave's syndrome, can mimic other serious medical problems on presentation. With an acutely ill patient, the physician is faced with a diagnostic dilemma and must maintain a high index of suspicion for the condition.

Spontaneous esophageal rupture, or Boerhaave's Syndrome, can mimic other serious medical conditions on presentation. Retrospective diagnosis of the condition may seem uncomplicated; however, with an acutely ill patient, the physician is faced with a diagnostic dilemma and must maintain a high index of suspicion. Boerhaave's Syndrome should be strongly suspected in any patient who presents with abdominal pain and a left sided pleural effusion. The following case demonstrates the ambiguous presentation, hospital course, and successful treatment of such a patient.

\section{Report of case}

A 56-year-old male construction worker was admitted to the Osteopathic Medical Center of Philadelphia (OMCP) with chief complaints of dyspnea, pleuritic chest pain, and hematemesis. The patient stated that he done some heavy lifting at work earlier in the day and had experienced dyspnea and chest pain soon after. On returning home, he had a single episode of hematemesis. The dyspnea and chest pain continued to increase, which prompted him to seek medical treatment.

He denied a history of similar problems, and his past medical and surgical histories were unremarkable. He stated that he was allergic to aspirin and denied taking any medications prior to admission. A 42-pack-year history of cigarette use and an alcohol intake of a fifth of whiskey per week for many years were elicited. Alcohol had been consumed on the evening prior to admission, but the patient denied excessive intake.

On arrival at the emergency room, he was awake, alert, oriented to person, place, and time, diaphoretic, and in moderate distress. Vital signs were as follows: blood pressure, $120 / 80 \mathrm{~mm} \mathrm{Hg}$; pulse rate, 116 beats per minute; respiratory rate, 26 per minute; oral temperature, $99^{\circ} \mathrm{F}$. No signs of trauma were visible. Auscultation of the heart revealed a regular rhythm, with tachycardia and without $S_{3}$ or $S_{4}$ sounds or murmur. The lungs were clear to auscultation bilaterally. The abdomen was soft, with decreased bowel sounds throughout and moderate epigastric tenderness on palpation. No masses or gross blood were seen on rectal examination. The extremities were without edema; all pulses were intact, equal, and symmetric. Neurologic examination findings were within normal limits.

Placement of a nasogastric tube recovered $500 \mathrm{~mL}$ of gross blood. The CBC (hemoglobin, $14.6 \mathrm{~g} / \mathrm{dL}$; hematocrit, $43.3 \%$; WBC, $9,400 / \mathrm{cu} \mathrm{mm}$ ), serum electrolyte values, and chest and abdominal $x$-ray films were within normal limits. Serial creatinine phosphokinase and lactic dehydrogenase isoenzyme determinations were unremarkable initially and on subsequent testing. An ECG showed sinus tachycardia without ST or T wave changes.

The patient continued to complain of dyspnea and sharp bilateral chest pain on deep inspiration or light palpation of the chest wall. A regimen of oxygen $(2 \mathrm{~L} /$ min), Zantac ( $50 \mathrm{mg}$, intravenously, every 6 hours), and nitroglycerin paste ( 1 inch every 6 hours) was begun, and he was admitted to the cardiac step-down unit for monitoring. The diagnosis was upper gastrointestinal bleeding. Atrial fibrillation developed after arrival to 
the unit, and digoxin ( $0.25 \mathrm{mg}$ intravenously/day) was added to the drug regimen.

$A$ repeat $\mathrm{CBC}$ (day 3 ) revealed hemoglobin and hematocrit levels of $10.9 \mathrm{~g} / \mathrm{dL}$ and $32.9 \%$, respectively, and a WBC elevated to $15,600 / \mathrm{cu} \mathrm{mm}$. Four units of packed red blood cells were transfused, and the hemoglobin and hematocrit values stabilized at $11.0 \mathrm{~g} / \mathrm{dL}$ and $32.7 \%$, respectively.

Serial chest roentgenograms showed the development of bilateral pleural effusion, which was thought to be the result of congestive heart failure secondary to recurrent atrial fibrillation. Right-sided thoracentesis recovered $60 \mathrm{~mL}$ of straw-colored fluid. Analysis showed the fluid to be exudative in nature, and no organisms were cultured.

Barium swallow and an upper gastrointestinal roentgenographic series were ordered. With the initial swallow of barium, extravasation into the mediastinum was noted. The procedure was stopped immediately, nothing by mouth (NPO) status was instituted, and the patient was monitored while preparations for surgery were made. The patient was in no acute distress, but he complained of mild chest wall discomfort.

Right-sided thoracotomy was performed on the fifth hospital day and an approximately 2-cm long perforation in the distal portion of the esophagus was repaired. Closure of the proximal esophagus was effected using a TA 55 stapling device. A nasogastric tube was placed in the proximal esophagus for decompression. An empyema at the right lung base was drained. Gastrostomy and jejunostomy tubes were placed. The patient tolerated the procedure well and was returned to the cardiac care unit in stable condition. Postoperatively, the patient's mediastinitis was treated with clindamycin, gentamycin, and piperacillin.

Esophagotomy was performed two weeks after the initial surgical repair. Following the procedure, the patient was discharged to home care. He returned to OMCP three weeks later for esophageal reanastomosis. The procedure was tolerated well, and recovery was uneventful.

\section{Discussion}

A relatively uncommon condition, spontaneous rupture of the esophagus is associated with high morbidity and mortality. With prompt surgical intervention, the survival rate is approximately $70 \% .^{1,2}$ In patients who remain untreated for longer than 48 hours, mortality approaches $90 \%{ }^{3}$ Cases of survival without surgical treatment have been reported, ${ }^{4,5}$ but they are rare.

In our patient, approximately 96 hours elapsed between the initial event and the surgical repair. His excellent physical condition contributed to his survival. More important, the patient had not eaten breakfast or lunch on the day of the esophageal tear, eliminating particulate matter from entering mediastinum. His only ingestion prior to arrival at the emergency room was 8 ounces of water, which he immediately regurgitated.

In the emergency room, prompt placement of a nasogastric tube and evacuation of the stomach con- tents $(500 \mathrm{~mL}$ blood), prevented reflux into the esophagus. Low pressure intermittent nasogastric suction, the patient's NPO status, and a left-sided thoracentesis on the fourth hospital day all helped to retard the development of the mediastinitis which occurred during the hospital stay.

It is not likely that the patient would have survived without surgical intervention. There was extensive tissue breakdown at the site of rupture, necessitating a two-part surgical repair over several weeks. The mediastinitis resolved after treatment with a 10-day course of intravenous antibiotic therapy.

The earliest recorded case of rupture of the esophagus was by Boerhaave in $1724 .^{6} \mathrm{He}$ gave a detailed account of the death of Baron John Van Wassener, grand admiral of Holland, who had attempted to induce vomiting following a large meal. While vomiting, the Baron experienced a sudden, sharp chest pain. He became dyspneic and developed subcutaneous emphysema and shock. Death occurred 18 hours later. Postmortem examination revealed bilateral empyema, mediastinal emphysema, and a recent tear of the lower one third of the esophagus.

Over the next 200 years, numerous reports of Boerhaave's syndrome appeared in the literature. The outcome was universally fatal, with postmortem discovery a common event. Surgical repair was not successful until Barrett's ${ }^{7}$ attempt in 1947. Since then, it has been considered the treatment of choice.

Over 300 cases of Boerhaave's syndrome have been reported in the world literature to date. ${ }^{8} \mathrm{~Pa}$ tients usually are in their fifth to seventh decades, and there is a male:female predominance that varies from $2: 1$ to $5: 1{ }^{1,9}$

\section{Etiology}

Classically, a large meal or an alcoholic binge precedes the rupture. There have been reports of esophagus rupture following asthma attacks, ${ }^{10}$ defecation, ${ }^{11}$ strenuous lifting, ${ }^{11}$ seizure,${ }^{11}$ during labor, ${ }^{11}$ with Antabuse therapy ${ }^{12,13}$ and with hemodialysis. ${ }^{14}$

Many theories have been postulated, but the etiology of Boerhaave's syndrome remains unknown. Vomiting is seen in approximately half of the cases. Some patients have prolonged vomiting, which may result in fatigue of the vomiting center in the sensory nucleus of the vagus nerve in the fourth ventricle. ${ }^{15}$ Discoordination of the vomiting reflex results, and failure of the upper and lower esophageal sphincters to relax simultaneously at the moment of greatest propulsive force results in an esophageal tear. Transmission of increased abdominal pressure to the esophagus has been proposed as a mechanism when vomiting has been absent.

Cadaveric studies ${ }^{16}$ demonstrated that it is the 
sudden increase in pressure rather than the total pressure that is responsible for the rupture. The initial damage occurs in the muscularis layer. The mucosal layer is able to herniate through the rent and rupture after further insults.

An esophagus with pre-existing damage will rupture more readily than a normal one. While strictures, webs, neoplasm, esophagitis, and hiatal hernia may aid in the formation of a tear, many tears have been reported ${ }^{17,18}$ in nondiseased organs. External sources of pressure, such as blunt chest trauma, ${ }^{19,20}$ bomb blasts, ${ }^{11}$ biting an inner tube,${ }^{11}$ and accidental release of pressurized air into the mouth, ${ }^{11}$ have been reported less frequently.

The majority of tears seen in Boerhaave's syndrome are in the lower one third of the esophagus, just above the cardia. Most often they are on the left side. ${ }^{21}$ In most instances, the tears are linear, with nonragged edges, and run longitudinally, with lengths from $0.6 \mathrm{~cm}$ to $8.9 \mathrm{~cm}$ reported. ${ }^{22}$ Because the mucosal layer ruptures after herniating through the muscularis, mucosal tears tend to be longer than the muscular tears. Failure to realize this may result in incomplete surgical repair and subsequent complications. The reflux of acid, pepsin bile, or gastric contents into the pleural cavity will interfere with esophageal healing and cause a severe mediastinitis. $9,18,23$

\section{Diagnosis}

The majority of patients present with sudden onset of severe chest pain that is unresponsive to analgesics. A classic triad of violent vomiting, hematemesis, and chest pain is present in almost $50 \%$ of patients. ${ }^{24}$ Significant hematemesis is not unusual; small amounts of blood are seen in the vomitus. Massive hematemesis is thought to be the result of a diffuse submucosal hemorrhage. ${ }^{2}$

A normal chest x-ray film, normotensive blood pressure, and normal respiration may be present early in the disease. However, the majority of patients present with complaints of dyspnea, with shallow, tachypneic respirations. They are anxious and may be tachycardic, and may have cool, clammy skin because of bacteremia and impending circulatory collapse. Pneumothorax or hydropneumothorax, if present, will contribute to the respiratory distress. Hamman's sign, a mediastinal "crunch" sound, is uncommon and may be mistaken for pericarditis. ${ }^{1}$

A lengthy differential diagnosis is the norm when one is dealing with Boerhaave's syndrome. The infrequent occurrence of the condition and its ambiguous symptoms may result in its premature exclusion from consideration. A high index of suspicion is needed when dealing with any chest or abdominal pain of sudden onset.
With hematemesis and abdominal pain, the physician would suspect perforated peptic ulcer. With Boerhaave's syndrome, abdominal findings decrease with time, as chest findings increase. With acute presentations, myocardial infarction, pulmonary embolus, and dissecting aortic aneurysm must be ruled out. ${ }^{25}$

Acute pancreatitis, ${ }^{26}$ which can present with vomiting, abdominal pain, and left pleural effusion, must be considered. With pancreatitis, the serum amylase value is elevated, while in Boerhaave's syndrome, the serum amylase is almost always nonelevated, and the pleural fluid amylase value is high. ${ }^{27}$

Coronary thrombosis, spontaneous pneumothorax, ruptured diaphragm, incarceration of a diaphragmatic hernia, renal colic, mesenteric artery thrombosis, and acute cholecystitis also should be considered in the differential diagnosis. Boerhaave's syndrome has been seen as a complication of acute myocardial infarction, ${ }^{28}$ and as the cause of unilateral proptosis secondary to orbital emphysema. ${ }^{29}$

Laboratory studies often are not helpful on admission. Patients may present with leukocytosis or a left shift, but no other abnormal laboratory values. When pleural effusion is present, a $\mathrm{pH}$ value $<6$ is considered to be a relatively certain sign of esophageal rupture. ${ }^{30}$

The most useful study is roentgenography. Erect films demonstrate unilateral effusion in up to $90 \%$ of patients and bilateral effusion in approximately $7 \% .{ }^{1}$ Free air in the mediastinum and subcutaneous air in the neck also may be seen. The V-sign of Nacliero ${ }^{31}$ is caused by air in the fascial planes and pleura behind the heart. It is an early, subtle finding and may go unnoticed.

The test of choice for confirming an esophageal tear is the esophagogram. Multiple views must be obtained to avoid missing a tear. Lack of extravasation from the esophagus does not exclude a tear, because barium may be too viscous and watersoluble contrast medium may pass by the lesion too rapidly for extravasation..$^{5,32}$

\section{Treatment}

Once the diagnosis is made, the accepted treatment for Boerhaave's syndrome is correction of electrolyte imbalances, vigorous volume replacement, and surgical repair as soon as possible.

The most common surgical procedure is exposure of the esophagus by thoracotomy on the side of the pleural effusion. ${ }^{21}$ The rupture is closed in two layers, and the pleural space is drained. The mediastinum is incised to allow for drainage into the pleural cavity; chest tubes are placed for pleural cavity drainage. 
Delays in initiating surgery allow tissue breakdown at the damaged site. Leakage, fistula formation, and empyema occur more often as the time between onset and surgical repair increases. To strengthen repairs and prevent subsequent tissue breakdown, pericardium, intercostal muscles, rhomboid muscle flaps, and stomach, lung, and diaphragmatic flaps have been used to buttress the primary repair. ${ }^{33-36}$

With very long delays in initiating surgery, suture and flaps may be ineffective. In these cases, cervical esophagostomy is performed. $3,24,37$ The continuity of the esophagus is maintained, the damaged area is repaired and drained, and the distal esophagus is occluded to prevent gastric reflux. Supplemental feeding via gastrostomy or hyperalimentation is used to provide the patient's nutritional needs while healing occurs. This approach is a multistep procedure and requires a prolonged period of time as well as multiple surgeries.

Within the last few years, several authors ${ }^{38-41}$ have suggested the nonsurgical management of Boerhaave's syndrome. If the patient has successfully weathered the initial mediastinitis and has survived the first 24 hours, nonsurgical management may yield good results.

\section{Comment}

The ambiguous initial presentation of the patient with Boerhaave's syndrome serves to reinforce the fact that this syndrome must be considered in the differential diagnosis of the acutely ill patient who presents with sudden onset of chest or abdominal pain. Early recognition and treatment greatly improve the patient's chance of survival.

\footnotetext{
1. Curci JJ, Horman MJ: Boerhaave's syndrome: The importance of early diagnosis and treatment. Ann Surgery 1976;183:401-408.

2. Bruno MS, Grier WR, Ober WB: Spontaneous laceration and rupture of the esophagus and stomach: Mallory Weiss syndrome, Boerhaave's syndrome, and their variants. Arch Intern Med 1963;112:574-583.

3. Symbas PN, Hatcher CR Jr, Harlaftips N, et al: Spontaneous rupture of the esophagus. Ann Surg 1978;187:634-640.

4. Larrieu AJ, Kieffer R: Boerhaave's syndrome: Report of a case treated non-operatively. Ann Surg 1975;181:452-454.

5. Sanderson RG: Spontaneous rupture of the esophagus: Report of survival without surgical management. Am J Surg 1965;109:506-508.

6. Derbes VJ, Mitchell RE Jr.: Herman Boerhaave's "Atrocis, nec descripti prius morbi historia." First translation from original Latin, 1724 of classic case report of rupture of the esophagus, with annotations. Bull Med Libr Assoc 1955;43:217-240.

7. Barrett NR: Report of a case of spontaneous perforation of the esophagus successfully treated by operation. $B r J$ Surg 1947;35:216-218.

8. Abbott OA, Mansour KA, Logan WD Jr, et al: Atraumatic so-called spontaneous rupture of the esophagus. A review of 47 personal cases with comments on a new method of surgical therapy. $J$ Thorac Cardiovasc Surg 1970;59:67-83.

9. Marston EL, Valk HL: Spontaneous perforation of the esophagus: Review of the literature and report of a case. Ann Int Med 1959;51:590. 607.

10. Raffle EJ: Spontaneous rupture of the esophagus and bronchial asthma. Lancet 1958;1:938-940.

11. Mackler SA: Spontaneous rupture of the esophagus: An experimen tal and clinical study. Surg Gynecol Obstet 1952;95:345-356.
}

12. Fernandez D: Another esophageal rupture after alcohol and disulfiram (correspondence). $N$ Engl J Med 1972;286:610.

13. Mayer JH, Herlocher J, Parisian J: Esophageal rupture after mushroom-alcohol ingestion (correspondence). N Engl J Med 1970;285:1323.

14. Klausner JM, Epstein L, Peer G, et al: Perforation of the esophagus (Boerhaave's syndrome) during hemodialysis. Nephron 1985;40:372373.

15. Flipse ME: Spontaneous rupture of the esophagus. Dis Chest 1951;19:165-189.

16. Burt CAV: Pneumatic rupture of the intestinal canal with experimental data showing the mechanism of perforation and the pressure required. Arch Surg 1931;22:875-902.

17. Alt RE, Bixby FE, Rowell RJ: Spontaneous rupture of the normal esophagus. N Engl J Med 1953;249:1062-1063.

18. Wichern WA: Perforation of the esophagus. Am J Surg 1970;119:534536.

19. Worman LW, Hurley JD, Demberton AH, et al: Rupture of the esophagus from external blunt trauma. Arch Surg 1962;85:333-338.

20. Goudarzi HA, Hall WW, Mason LB: Rupture of the cervical esophagus from blunt trauma. South Med $J$ 1976;76:1563-1564

21. Sealy WC: Rupture of the esophagus. Am J Surg 1963;105:505-510. 22. Bennett DJ, Deveridge RJ, Wright TS: Spontaneous rupture of the esophagus: A review with reports of six cases. Surgery 1970; 68:766. 770 .

23. Urschel HC, Kazzok MA, Wood RE, et al: Improved management of esophageal perforation: Exclusion and diversion in continuity. Ann Surg 1974;179:587-591.

24. Patton AS, Lawson DW, Shannon JM, et al: Re-evaluation of Boerhaave's syndrome: A review of fourteen cases. Am J Surg 1979;137:560 565 .

25. delCastillo J, Boyar C, Hess F, et al: Atraumatic panmural rupture of the esophagus: Boerhaave's syndrome. Ann Emerg Med 1983;12:385390.

26. Levine $\mathrm{PH}$, Kelley ML Jr.: A report of rare spontaneous perforation of the esophagus simulating acute pancreatitis in man. JAMA 1965;191:343-345.

27. Sherr HP, Light RW, Merson MH, et al: Origin of the pleural fluid amylase in esophageal rupture. Ann Intern Med 1972;76:985-986.

28. Michalik R, Hatcher CR Jr, Miller JJ: Myocardial infarction complicated by Boerhaave's syndrome. South Med $J$ 1984;77:504-506.

29. Schneider SM, Goodman D: Spontaneous rupture of the esophagus presenting with unilateral proptosis. Ann Emerg Med 1984;13:374-377 30. Dye RA, Laforet EG: Esophageal rupture: Diagnosis by pleural fluid pH. Chest 1974;66:454-456.

31. Nacliero EA: The "V-sign" in the diagnosis of spontaneous rupture of the esophagus (an early roentgen clue). Am J Surg 1957;93:291-298. 32. Tesler MA, Eisenberg MM: Spontaneous esophageal rupture. Int Ab. str Surg 1963;117:1-10.

33. Slater G, Sicular AA: Esophageal perforation after forceful dilatation in achalasia. Ann Surg 1982;195:186-188.

34. Lucas AE, Snow N, Tobin GR, et al: Use of the rhomboid major muscle flap for esophageal repair. Ann Thorac Surg 1982;33:619-623.

35. Nissen R: Bridging of esophageal defect by pedicled flap of lung tissue. Ann Surg 1949;129:142-147

36. Rao KVS: Management of perforation of the thoracic esophagus: A new technique utilizing a pedicle flap of diaphragm. Am J Surg 1974;127:609-612

37. Triggiani E, Belsey R: Esophageal trauma: Incidence, diagnosis and management. Thorax 1977;32:241-250.

38. Asplund CM, Hill LD: Delayed lower esophageal perforation: Management with celestin tube. Ann Otol Rhinol Laryngol 1985;94:114-116. 39. Walker WS, Cameron EW, Walbaum PR: Diagnosis and management of spontaneous transmural rupture of the esophagus (Boerhaave's syndrome) $B r J$ Surg 1985;72:204-207.

40. Hutter JA: The management of spontaneous esophageal perforation by thorascopy and irrigation. Br J Surg 1985;72:208-209:

41. Lyons WS: Ruptures and perforation of the esophagus: The case for conservative supportive management. Ann Thorac Surg 1978;25:346. 350 .

From the Department of Cardiology, Philadelphia College of Osteopathic Medicine. Dr Zawisza is a family practice resident.

Reprint requests to Dr Zawisza, Good Samaritan Hospital, Pottsville, PA 17901. 